\title{
Data Mining from Heterogeneous Data Sources
}

\author{
U Mahender \\ Assistant Professor, CMR Engineering College, Hyderabad, TS.
}

\begin{abstract}
Information Retrieval from heterogeneous information structures is highly troublesome and data is secured additionally, as addressed in different data models in different information systems. Information facilitated from heterogeneous data sources is combed into single data source faces noteworthy trial of information change were in different arrangements and necessities in data change are used as a piece of data joining with the ultimate objective of consolidating information structures, and same is not monetarily canny. This paper presents idea of Information mix in perspective of interest criteria from heterogeneous data sources into single data source [1]. Every segment of information source, for instance, substance, field, and association is mapped to a portion of new single content source-made every time heterogeneous information structures are looked for and result is saved into new substance record. This approach grants us to make new substance archive and delete existing record, modifying wrapper, making changes later also, administering data recuperation in a fundamental bound together style. This plan is adequately versatile to merge variety of data models also, address limits by various traditions. It is possible to pick reliably tied information from all open legacy data sources.
\end{abstract}

Keywords: Data Mining, Heterogeneous data, Knowledge Discovery, Heterogeneous Data sources, Semantics, Databases, Big Data

\section{Introduction}

In a universe of wide scale information sharing, coordination strategies are turning out to be increasingly testing. Data is anticipated that would be discovered divided and circulated among various self-sufficient sources, making information recovery a confused technique. The circumstance is further compounded on the off chance that we consider the noteworthy heterogeneity, saw between these sources: Shared information is put away in various frameworks, depicted by different configurations and involves distinctive semantics. Information coordination methodologies [3] are attempting to explain these weights, with the goal that client questions will have the capacity to recover the normal answers, consolidated accurately from different sources Associations, both administrative and business, need to oversee vast measure of data put away in some type of databases or documents. One of the fundamental issues to manage data overseeing is the feeble interoperability between different databases and data frameworks[2]. Particularly this issue is complex when we need sort out a joint effort between the data frameworks of different offices inside the association. Information recovery from various self-ruling sources has turned into an interesting issue amid the most recent years. For example, there are such information sources as worker information source, understudy information source, library information source and so on inside a similar venture (discussing scholastic foundation). When somebody needs bit of data we have to execute $\mathrm{n}$ inquiries and perhaps give client $\mathrm{n}$ such outcomes, recovered from $\mathrm{n}$ information sources. Heterogeneous information sources are looked in light of client criteria and aftereffect of $\mathrm{n}$ sources is coordinated into single source, this information source is made each time heterogeneous data frameworks are to be looked and structure of this single information source is rapid and not static in that capacity structure of this source is variable and is characterized a new every time.

\section{Centralized Data warehouse system}

Information stockpiling [4] is sorted out in a totally decentralized way and data recovery may include questioning numerous information sources. In extensive ventures for instance, where choices are normally mentioned in light of information objective facts, each office may keep its own particular database framework (HRM, Finance, Sales and so on). In this manner collected data for the entirety venture requires information mixes from different sources. The decentralization is further improved on the off chance that we additionally consider conceivable accomplices, merchants or contenders, whose information may be of organization's advantage. Another zone, where this decentralization compounds data recovery is expansive scale logical activities. Here the volume of information, as well as the many-sided quality needs to be considered. Researchers these days, other than significant space information, oblige access to information and results gave by others. In this manner, questioning separately unique information sources prompts to huge wastefulness in their work. At last, for a powerful inquiry in Enterprise, client is required to gaze upward for data in different information sources and gather the information separately.

Notwithstanding the decentralization [6], the adequacy of data recovery is further worsened by the assortment of heterogeneity display in the information sources. In each of these sources, information is organized using an alternate framework (working frameworks, SQL Vendor Implementations and so forth), in light of different reasonable models, and on various organizations "framework level data heterogeneity" is these days much less demanding than before, much interest is laid on the alleged "semantic heterogeneity", which seems each time there is a more than one approach to structure an assortment of data. Semantic heterogeneity [5] is by all accounts an unavoidable weight in information sharing and control, since people tend to display their information as per their own particular comprehension of the truth. This obviously is fundamentally diverse for every person. In that sense, 


\section{International Journal of Science and Research (IJSR) \\ ISSN (Online): 2319-7064}

Index Copernicus Value (2015): 78.96 | Impact Factor (2015): 6.391

heterogeneity is to be found in information models, conceptual patterns, and obviously the brain of the clients.

\section{Proposed Approach}

The basic principle of information joining is to combine(integrate) chosen data sources from specific space, in a way that an entire new data Source is created. The enduser, when questioning for information, has the dream of interacting with one single framework, which presents him a brought together sensible perspective of the information accessible. The principal endeavors to address data joining issues in ventures where construct fundamentally in light of information warehousing systems, however our proposed design, is depicted graphically beneath. Customary arrangements endorse formation of new information source on Information coordination from heterogeneous information sources, which is not savvy.

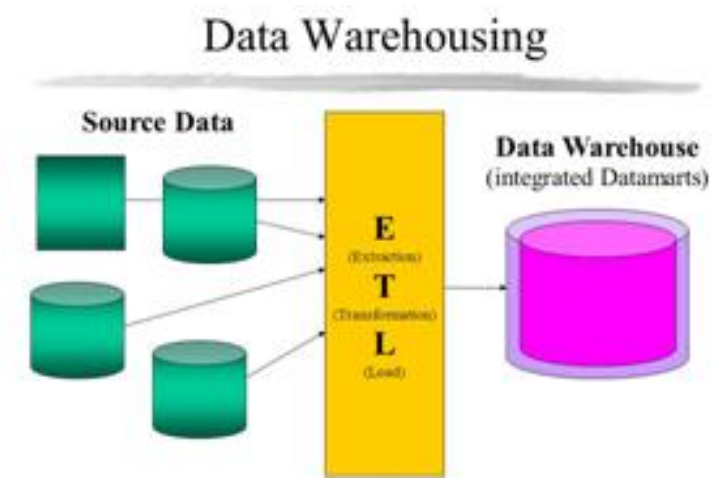

The mapping gives the client a bound together perspective of all information, on which questions can be postured. This specific blueprint is not intended to store any information; it is absolutely a consistent outline. A client question (Q) [7] is reformulated over the source compositions consequently in view of the arrangement of standards $M$ (Source portrayals) and sought locally on the independent content sources. Henceforth, the reformulation of Q results in an arrangement of source-particular questions Qi, whose blend will yield the response to the underlying inquiry $\mathrm{Q}$.

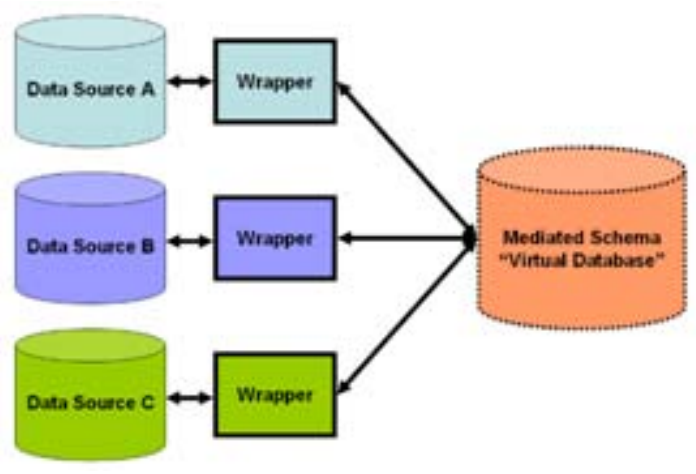

\section{Heterogeneous Data Encapsulating}

There are two fundamental settings in which the issue of noting questions utilizing sees has been considered. In the first setting, where the objective is inquiry advancement or upkeep of physical information freedom, we look for an expression that utilizes the perspectives and is equal to the first inquiry. Here it is typically expected that the quantity of perspectives is on an indistinguishable request from the extent of the construction. The second setting is that of information combination, where sees depict an arrangement of self-governing heterogeneous information sources. A client represents an inquiry as far as an intervened outline, and the information mix framework [8] needs to reformulate the question to allude to the information sources. In an ensuing stage, the questions over the sources are streamlined and executed. The reformulation issue can be fathomed by calculations for noting questions utilizing sees, however in this unique circumstance, we for the most part can't discover a reworking that is comparable to the client inquiry on account of the information sources restricted scope. In a few information coordination applications, the quantity of information sources might be very extensive - for instance, information sources might be an arrangement of sites, a substantial arrangement of providers and purchasers in an electronic commercial center, or an arrangement of companions containing parts of a bigger information set in a distributed situation. Consequently, the test in this setting is to build up an arrangement that scales up in the number of perspectives. All things considered, client question postured as far as an intervened mapping, and the information combination framework needs to reformulate the inquiry to allude to the information sources. Since there are $n$ heterogeneous information source, however client craved outcome might be available in $m$ sees where $n>m$, all things considered it is the duty of Wrapper to recognize $m$ sources and plan resultant $\mathrm{m}$ queries. In an ensuing stage, the questions over the sources are upgraded and executed. To minimize information recovery, Wrapper produces extremely specific SQL, returning just the information that is required. To maintain a strategic distance from recovering columns that are not required, the conditions in Where provisions and predicates are changed over to Where statements in the produced SQL. To abstain from recovering segments that are not required, the created SQL indicates the sections really required by the client [9].

\section{Transformation of knowledge}

Wrapper-sends inquiries to an information source, gets replies back, possiblyapplies fundamental changes and makes new content source, this recently made content source is characterized in agreement to the outcome created therefore of executing question on heterogeneous information sources, and changed information is put away in this content source, at last client is given outcome from this content source-source content document is tab isolated, all things considered client can be given outcome in coveted organization e.g .pdf,.doc,.xls and so on. It is normal that applications need to manage information which is not accessible in a solitary organization; and that is the unique circumstance where managing a solitary question dialect, information model and interface which covers heterogeneous information sources gets to be essential. Consider a situation, for instance, where a rundown of sold ITEMs is accessible in a XML report, as yet insights about the individual who's putting forth the ITEM are accessible in a USERS table facilitated on a social database, including data about the client id, name, address and email. Presently consider the need of making an application that given a 


\section{International Journal of Science and Research (IJSR) \\ ISSN (Online): 2319-7064}

Index Copernicus Value (2015): 78.96 | Impact Factor (2015): 6.391

client's email address recovers every one of the things that are being sold by that client. The wrapper expending the outcome knows about the physical source of the information returned thus of execution of inquiries regardless of the possibility that the outcome blends data put away in a social database and in a XML report. Since information got by the wrapper are in various arrangements is changed into nonexclusive configuration, separated information is changed and spared into content source, before sparing in content arrangement content source is made as per information recovered subsequently of execution of $n$ questions on $\mathrm{n}$ heterogeneous information sources, definition incorporates segment definition. Removed, refined, cleaned, changed, spared information in temp content source is passed onto client.

\section{Query Request Processing}

Another issue that must be reclassified in Data Integration situations is inquiry handling. In a customary DBMS, inquiry handling is included particularly from an inquiry streamlining and a question execution stage. Question is improved at aggregate time, creating a question execution arrange at run time, which takes after entirely the directions of the improvement. However, in Data Combination Applications, a streamlined inquiry execution arrange can't be built amid assemblage, since properties of the information sources are typically obscure heretofore (cardinalities, requesting data, histograms and other selectivity estimation helps, conditions and uniqueness limitations). Furthermore, the working environment of every information source is additionally obscure (CPU speed, circle get to time etc. A few works examined expansions to question analyzers that attempt to make utilization of appeared perspectives in inquiry preparing. At times, they altered the System-R style join list segment, and in others they fused view rewritings into the rework period of the streamlining agent. These works demonstrated that considering the nearness of appeared perspectives did not contrarily affect the execution of the enhancer. In any case, in these works the quantity of perspectives had a tendency to be moderately little. We consider the issue of finding the most proficient changing of the inquiry utilizing an arrangement of perspectives, with regards to question enhancement, where question execution plan is being altered at run time. Planning based Methods that safeguard the intelligent structure of the inquiry arrange, however re-plan the request in which operations are handled by the CPU. Excess Computation Methods that utilization a few question arrangements to prepare similar information. The most effective arrangement is at long last executed and the rest are relinquished.

\section{Conclusion}

In this paper we have examined how Single Wrapper can be valuable in giving information administrations which achieve information combination errands crosswise over heterogeneous information sources. Keeping in mind the end goal to prevail in that undertaking, Wrapper execution must be enhanced to bargain with the idiosyncrasies of the different bolstered information sources. Wrapper actualizes an assortment of systems when managing social databases and XML reports; those incorporate the capacity to push SQL to the social motor, to minimize the sum of information recovered from the database, change, refine, clean $\&$ spare information into content source at long last is passed onto client. In spite of the fact that Data Integration was thought to be "a territory of scholarly curiosity"[1] at its initial years, the appearance of data sharing these days is calling for compelling incorporation approaches acknowledged by and by. Clients are not trading off with low gauges of data precision and will locate the correct data at the opportune time. The examination group, up to this point, has indicated superb advance in managing the most critical issues introduced in transit of incorporating information, be that as it may, additionally challenges emerge continually: The development of semi and unstructured information (XML) for instance suggests that information sources are considerably more unpredictable and hard to handle. Adapting to semantic heterogeneity in such situations appears practically unimaginable. Nonetheless, research is getting significantly more exceptional and promising thoughts are relied upon to create.

\section{References}

[1] Dunham, M. H., Sridhar S., "Data Mining: Introductory and Advanced Topics", Pearson Education, New Delhi, ISBN: 81-7758-785-4, 1st Edition, 2006.

[2] Baazaoui, Z., H., Faiz, S., and Ben Ghezala, H., "A Framework for Data Mining Based Multi-Agent: An Application to Spatial Data, volume 5, ISSN $1307-$ 6884," Proceedings of World Academy of Science, Engineering and Technology, April 2005.

[3] Chen, H., Chung, W., Qin, Y., Chau, M., Xu, J. J., Wang, G., Zheng, R., Atabakhsh, H., "Crime Data Mining: An Overview and Case Studies", A project under NSF Digital Government Programme, USA, "COPLINK Center: Information and Knowledge Management for Law Enforcement,", July 2000 - June 2003

[4] Ghani, R., Probst, K., Liu, Y., Krema, M., Fano, A., "Text Mining for Product Attribute Extraction", SIGKDD Explorations Volume 8, Issue 1.

[5] Onkamo, P. and Toivonen, H., "A survey of data mining methods for linkage dis-equilibrium mapping", Henry Stewart Publications 1473 - 9542. Human Genomics. VOL 2, NO 5, Page No. 336-340, MARCH 2006.

[6] Jadhav, S. R., and Kumbargoudar, P., "Multimedia Data Mining in Digital Libraries: Standards and Features".Proceedings of conference Recent advances in Information Science and Technology READIT - 2007, pp 54-59, Organized by Madras Library Association Kalpakkam Chapter \&Scintific information Resource Division, Indira Gandhi Center for Atomic research, Department of Atomic Energy, Kalpakkam, Tamilnadu,India. 12-13 July 2007.

[7] Dr. M.H.Dunham, "Data Mining, Introductory and Advanced Topics", Prentice Hall, 2002

[8] D Ramesh , B Vishnu Vardhan, "Data Mining Techniques and Applications to Agricultural Yield Data" International Journal of Advanced Research in Computer and Communication Engineering Vol. 2, Issue 9, September 2013

[9] Umamaheswari. K, S. Niraimathi "A Study on Student Data Analysis Using Data Mining Techniques" 


\section{International Journal of Science and Research (IJSR) \\ ISSN (Online): 2319-7064}

Index Copernicus Value (2015): 78.96 | Impact Factor (2015): 6.391

International Journal of Advanced Research in Computer Science and Software Engineering, Volume 3, Issue 8, August 2013

\section{Author Profile}

U Mahender, is working as Assistant Professor at CMR Engineering college, Medchal, Hyderabad. His areas of Interest Are C Programming and Data Mining. He has vast experience in teaching and research. 